\title{
Design of Impedance Matching Networks for RF Applications
}

\author{
Frederick Ray I. Gomez \\ New Product Introduction Department, Back-End Manufacturing \& Technology, STMicroelectronics, Inc. \\ 9 Mountain Drive, Light Industry \& Science Park II, Brgy. La Mesa, Calamba City, Laguna, Philippines 4027 \\ Email: frederick-ray.gomez [AT] st.com
}

\begin{abstract}
This technical paper presents a design and study of impedance matching for RF (radio frequency) circuit application of common-source amplifier topology. Input and output matching networks of the amplifier were designed and computed ensuring unconditional stability. Inductors and capacitors are key passive components that are crucial for impedance matching, and are specifically designed such that they would satisfy the gain requirements at a specific frequency of operation. Impedance matching is necessary in RF circuit design to provide maximum possible power transfer between the source or the generator and the load. Complex tradeoffs among technology specifications and design parameters exist and should be carefully handled when designing the impedance matching networks, to optimize the performance of the amplifier.
\end{abstract}

Keywords - Impedance matching, matching networks, RF, common-source amplifier, inductor, capacitor, s-parameters

\section{INTRODUCTION}

Impedance matching plays vital role in optimizing the performance of the RFIC (radio frequency integrated circuit) design. Matching provides maximum power transfer between the input or source and the output or the load, thus allowing the RF circuit to achieve the desired performance esp. the gain requirements. Inductors and capacitors are key passive components that are crucial for impedance matching, and are specifically designed such that they would satisfy the gain requirements at a specific frequency or range of operation [1] [2] [3]. Design tradeoffs between matching network parameters are inevitable, so it is crucial that inductors and capacitors be designed carefully for the specific requirements of the intended application.

\section{DESIGN METHODOLOGY}

For this particular study, actual S-parameters of a $300 \mu \mathrm{m} / 0.25 \mu \mathrm{m}$ transistor (in touchstone format) were initially provided, for RF circuit application of common-source amplifier circuit topology. Required values of S-parameters for a specific frequency of operation could then be determined using linear interpolation. Shown in Table 1 are the Sparameters of the transistor at frequency initially set to $2.6 \mathrm{GHz}$. Moreover, a $10-\mathrm{dB}$ gain requirement is set for this study.

Table 1: S-parameters of transistor at frequency of $2.6 \mathrm{GHz}$

\begin{tabular}{c|c|c}
\hline S-Parameters & Real & Imaginary \\
\hline$S_{11}$ & 0.599858625 & -0.53991373 \\
$S_{21}$ & -0.219423779 & 1.14183461 \\
$S_{12}$ & 0.067523223 & 0.03730980 \\
$S_{22}$ & 0.116580879 & -0.40044436 \\
\hline
\end{tabular}

Stability conditions of the two-port network in terms of S-parameters play an essential role in amplifier designs. Although stability is frequency dependent, we want to ensure that the amplifiers design exhibits unconditional stability esp. at higher frequencies.

There are several ways to check for the stability of the two-port network. Expressions of stability constants in Eq. (1) to (6) could be used to check for the stability of the design. Computed values are shown in Table 2. These can also be used to compute for the source/generator and load reflection coefficients which will be shown later. 


$$
\begin{aligned}
& \Delta=\operatorname{det}(S)=S_{11} S_{22}-S_{12} S_{21} \\
& K=\frac{1-\left|S_{11}\right|^{2}-\left|S_{22}\right|^{2}+|\Delta|^{2}}{2\left|S_{12} S_{21}\right|} \\
& B_{1}=1+\left|S_{11}\right|^{2}-\left|S_{22}\right|^{2}-|\Delta|^{2} \\
& B_{2}=1+\left|S_{22}\right|^{2}-\left|S_{11}\right|^{2}-|\Delta|^{2} \\
& C_{1}=S_{11}-\Delta S_{22}^{*} \\
& C_{2}=S_{22}-\Delta S_{11}^{*}
\end{aligned}
$$

Table 2: Stability constants

\begin{tabular}{c|c}
\hline Stability Constants & Values \\
\hline$\Delta$ & $0.382530255<-103.4315597^{\circ}$ \\
$K$ & 1.789571393 \\
$B_{1}$ & 1.331061024 \\
$B_{2}$ & 0.376280183 \\
$C_{1}$ & $0.652080692<-44.9832792^{\circ}$ \\
$C_{2}$ & $0.132947589<-103.4852039^{\circ}$ \\
\hline
\end{tabular}

To have unconditional stability, the Rollett stability factor $\mathrm{K}$ must be greater than unity, that is, $\mathrm{K}>1$, as well as one other condition [1] [2]. Hence, any of the following criteria is sufficient and necessary for unconditional stability:

$$
\begin{array}{ll}
K>1 \text { and }|\Delta|<1 & \text { Eq. (7) } \\
K>1 \text { and } B_{1}>0 & \text { Eq. (8) } \\
K>1 \text { and } B_{2}>0 & \text { Eq. (9) } \\
K>1 \text { and }\left|S_{12} S_{21}\right|<1-\left|S_{11}\right|^{2} & \text { Eq. (10) } \\
K>1 \text { and }\left|S_{12} S_{21}\right|<1-\left|S_{22}\right|^{2} & \text { Eq. (11) }
\end{array}
$$

Table 3 shows the condition values of all the unconditional stability criteria.

Table 3: Unconditional stability criteria

\begin{tabular}{c|c|c}
\hline Criteria & Values & Check Condition \\
\hline$K>1$ & $1.789571393>1$ & $\checkmark$ \\
$|4|<1$ & $0.382530255<1$ & $\checkmark$ \\
$B_{1}>0$ & $1.331061024>0$ & $\checkmark$ \\
$B_{2}>0$ & $0.376280183>0$ & $\checkmark$ \\
$\left|S_{12} S_{21}\right|<1-\left|S_{11}\right|^{2}$ & $0.089698963<0.34866279$ & $\checkmark$ \\
$\left|S_{12} S_{21}\right|<1-\left|S_{22}\right|^{2}$ & $0.089698963<0.82605321$ & $\checkmark$ \\
\hline
\end{tabular}

It can be observed that all of the conditions are met. Therefore, the two-port network in terms of S-parameters is unconditionally stable. Maximum power transfer is achieved when both the generator and load are conjugately matched to the two-port network, that is,

$$
\begin{aligned}
& \Gamma_{\text {in }}=\Gamma_{G}^{*} \text { and } \Gamma_{\text {out }}=\Gamma_{L}^{*} \\
& Z_{\text {in }}=Z_{G}^{*} \text { and } Z_{\text {out }}=Z_{L}^{*}
\end{aligned}
$$

Where

$\Gamma_{\text {in }}=$ input reflection coefficient of the two-port network

$\Gamma_{\text {out }}=$ output reflection coefficient of the two-port network 
$\Gamma_{G}=$ source or generator reflection coefficient

$\Gamma_{L}=$ load reflection coefficient

$Z_{\text {in }}=$ input impedance of the two-port network

$Z_{\text {out }}=$ output impedance of the two-port network

$Z_{G}=$ source or generator impedance

$Z_{L}=$ load impedance

Figure 1 shows the block/schematic diagram of a two port network impedance matching networks.

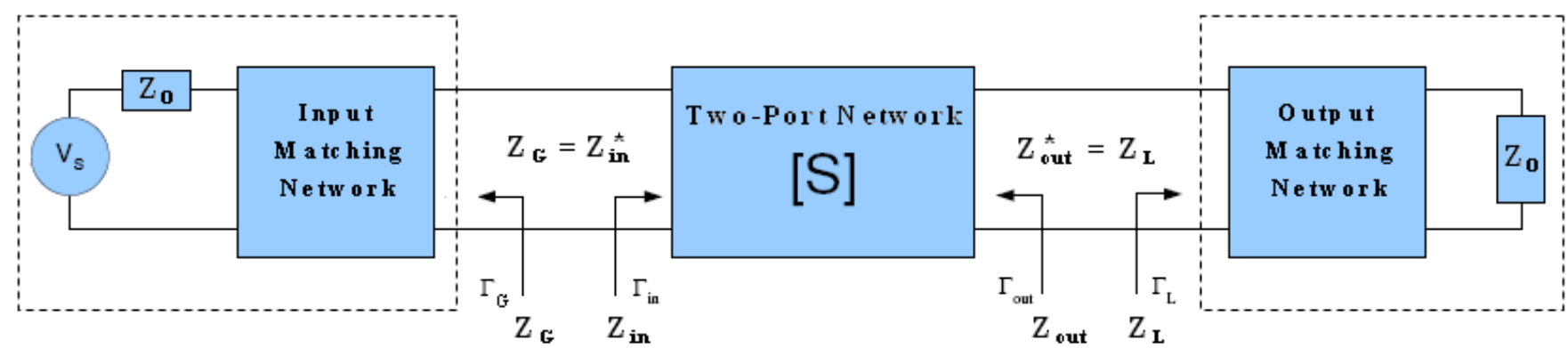

Figure 1: Two-port network with input and output matching networks

Through simultaneous conjugate matching, the following reflection coefficients can be obtained:

$$
\begin{aligned}
& \Gamma_{\text {in }}=\Gamma_{G}^{*}=S_{11}+\frac{S_{12} S_{21} \Gamma_{L}}{1-S_{22} \Gamma_{L}}=\frac{S_{11}-\Delta \Gamma_{L}}{1-S_{22} \Gamma_{L}} \\
& \Gamma_{\text {out }}=\Gamma_{L}^{*}=S_{22}+\frac{S_{12} S_{21} \Gamma_{G}}{1-S_{11} \Gamma_{G}}=\frac{S_{22}-\Delta \Gamma_{G}}{1-S_{11} \Gamma_{G}}
\end{aligned}
$$

Alternatively, the source/generator and load reflection coefficients in Eq. (16) and (17) could be derived using the expressions in Eq. (3) to (6).

$$
\begin{gathered}
\Gamma_{G}=\frac{B_{1}-\sqrt{B_{1}^{2}-4\left|C_{1}\right|^{2}}}{2 C_{1}} \\
\Gamma_{L}=\frac{B_{2}-\sqrt{B_{2}^{2}-4\left|C_{2}\right|^{2}}}{2 C_{2}}
\end{gathered}
$$

Using the expressions in Eq. (16) and (17), source/generator and load impedances could now be obtained.

$$
\begin{aligned}
& Z_{G}=\left(\frac{1+\Gamma_{G}}{1-\Gamma_{G}}\right) Z_{0} \\
& Z_{L}=\left(\frac{1+\Gamma_{L}}{1-\Gamma_{L}}\right) Z_{0}
\end{aligned}
$$

Table 4 shows the values of all the reflection coefficients as well as the impedances, assuming normalization impedance of $\mathrm{Z}_{0}=50 \Omega$. 
Table 4: Reflection coefficients and impedances

\begin{tabular}{c|c}
\hline$\Gamma$ and $\boldsymbol{Z}$ & Values \\
\hline$\Gamma_{\text {in }}$ & $0.577503798-\mathrm{j} 0.577166827$ \\
$\Gamma_{\text {out }}$ & $-0.096502369-\mathrm{j} 0.402419084$ \\
$\Gamma_{G}$ & $0.577503798+\mathrm{j} 0.577166827$ \\
$\Gamma_{L}$ & $-0.096502369+\mathrm{j} 0.402419084$ \\
$Z_{\text {in }}$ & $32.57933879-\mathrm{j} 112.810612 \Omega$ \\
$Z_{\text {out }}$ & $30.37350084-\mathrm{j} 29.4972738 \Omega$ \\
$Z_{G}$ & $32.57933879+\mathrm{j} 112.810612 \Omega$ \\
$Z_{L}$ & $30.37350084+\mathrm{j} 29.4972738 \Omega$ \\
\hline
\end{tabular}

For the input and output matching networks, L-network is used because it is the simplest and most widely used matching network for lumped elements, as shown in Figures 2 to 3.

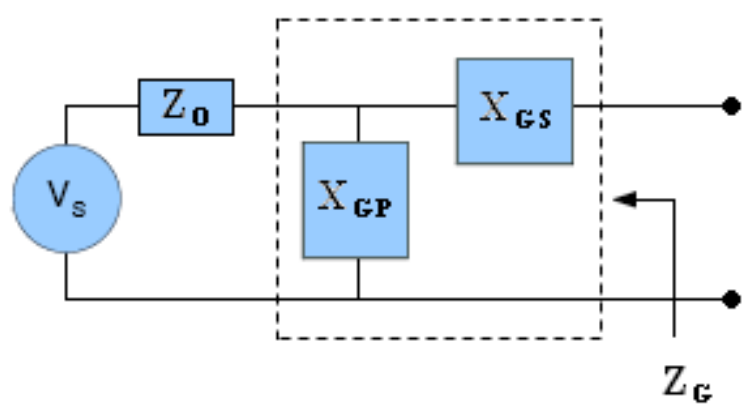

Figure 2: L-network of the input matching network

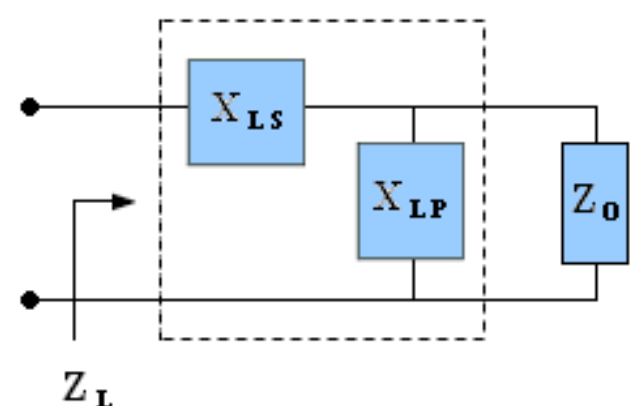

Figure 3: L-network of the output matching network

Where

$X_{G S}=$ series reactance of the L-network of the input matching network

$X_{G P}=$ parallel reactance of the L-network of the input matching network

$X_{L S}=$ series reactance of the L-network of the output matching network

$X_{L P}=$ parallel reactance of the L-network of the output matching network

The elements of the L-network for both the input and output matching network as shown in Figures 2 to 3 are arranged in such orientation given that the real components of $\mathrm{Z}_{\mathrm{G}}$ and $\mathrm{Z}_{\mathrm{L}}$ (or $\mathrm{R}_{\mathrm{G}}$ and $\mathrm{R}_{\mathrm{L}}$ ) are smaller than the real component of the normalization impedance which is $\mathrm{Z}_{0}=50 \Omega\left(\right.$ or $\mathrm{R}_{0}=50 \Omega$ ) [1] [2]. To check,

$$
\begin{aligned}
& R_{G}=32.57933879 \Omega<R_{0}=50 \Omega \\
& R_{L}=30.37350084 \Omega<R_{0}=50 \Omega
\end{aligned}
$$


For the L-network of the input matching network, the elements can be solved using the following equations given that $\mathrm{Z}_{0}=50 \Omega\left(\mathrm{R}_{0}=50 \Omega, \mathrm{X}_{0}=0\right)$ :

$$
\begin{aligned}
Q_{G}=\sqrt{\frac{R_{0}}{R_{G}}-1} \\
\text { or } X_{G P}= \pm \frac{R_{0}}{Q_{G}} \\
X_{G P 1}=+\frac{R_{0}}{Q_{G}} \\
X_{G P 2}=-\frac{R_{0}}{Q_{G}} \\
X_{G S}=-\left(-X_{G} \pm R_{G} Q_{G}\right) \\
\text { or } X_{G S 1}=-\left(-X_{G}+R_{G} Q_{G}\right) \\
X_{G S 2}=-\left(-X_{G}-R_{G} Q_{G}\right)
\end{aligned}
$$

Likewise, for the L-network of the output matching network, the elements can be solved using the following equations given that $\mathrm{Z}_{0}=50 \Omega\left(\mathrm{R}_{0}=50 \Omega, \mathrm{X}_{0}=0\right)$ :

$$
\begin{aligned}
Q_{L}=\sqrt{\frac{R_{0}}{R_{L}}-1} \\
X_{L P}= \pm \frac{R_{0}}{Q_{L}} \\
\text { or } X_{L P 1}=+\frac{R_{0}}{Q_{L}} \\
X_{L P 2}=-\frac{R_{0}}{Q_{L}} \\
X_{L S}=-\left(-X_{L} \pm R_{L} Q_{L}\right) \\
\text { or } X_{L S 1}=-\left(-X_{L}+R_{L} Q_{L}\right) \\
X_{G S 2}=-\left(-X_{L}-R_{L} Q_{L}\right)
\end{aligned}
$$

Table 5 summarizes the values obtained from the expressions Eq. (29) to (35).

Table 5: L-network elements

\begin{tabular}{c|c}
\hline $\boldsymbol{Q}$ and $\boldsymbol{Z}$ & Values \\
\hline$Q_{G}$ & 0.731242 \\
$X_{G P 1}$ & $68.376808 \Omega$ \\
$X_{G P 2}$ & $-68.376808 \Omega$ \\
$X_{G S 1}$ & $88.987228 \Omega$ \\
$X_{G S 2}$ & $136.633996 \Omega$ \\
$Q_{L}$ & 0.803848 \\
$X_{L P 1}$ & $62.200806 \Omega$ \\
$X_{L P 2}$ & $-62.200806 \Omega$ \\
$X_{L S 1}$ & $5.081593 \Omega$ \\
$X_{L S 2}$ & $53.912955 \Omega$ \\
\hline
\end{tabular}

Actual capacitor and inductor values at $\mathrm{f}=2.6 \mathrm{GHz}$ can be computed from the L-network reactances. Positive reactance denotes an inductive component while a negative reactance implies a capacitive component.

$$
j X_{G P 1}=j \omega L_{G P 1}
$$




$$
\begin{aligned}
& L_{G P 1}=\frac{X_{G P 1}}{\omega}=\frac{68.37680755}{2 \pi(2.6 G H z)}=4.185579582 n H \\
& -j X_{G P 2}=\frac{1}{j \omega C_{G P 2}} \\
& C_{G P 2}=\frac{1}{\omega X_{G P 2}}=\frac{1}{2 \pi(2.6 G H z)(68.37680755)}=0.895236877 p F \\
& j X_{G S 1}=j \omega L_{G S 1} \\
& L_{G S 1}=\frac{X_{G S 1}}{\omega}=\frac{88.98722805}{2 \pi(2.6 G H z)}=5.447214314 n H \\
& j X_{G S 2}=j \omega L_{G S 2} \\
& L_{G S 2}=\frac{X_{G S 2}}{\omega}=\frac{136.633996}{2 \pi(2.6 G H z)}=8.363836868 n H \\
& j X_{L P 1}=j \omega L_{L P 1} \\
& L_{L P 1}=\frac{X_{L P 1}}{\omega}=\frac{62.20080573}{2 \pi(2.6 G H z)}=3.807525268 n H \\
& -j X_{L P 2}=\frac{1}{j \omega C_{L P 2}} \\
& C_{L P 2}=\frac{1}{\omega X_{L P 2}}=\frac{1}{2 \pi(2.6 \mathrm{GHz})(62.20080573)}=0.9841261529 \mathrm{pF} \\
& j X_{L S 1}=j \omega L_{L S 1} \\
& L_{L S 1}=\frac{X_{L S 1}}{\omega}=\frac{5.081592616}{2 \pi(2.6 \mathrm{GHz})}=0.3110617629 \mathrm{nH} \\
& j X_{L S 2}=j \omega L_{L S 2} \\
& L_{L S 2}=\frac{X_{L S 2}}{\omega}=\frac{53.91295498}{2 \pi(2.6 G H z)}=3.300197416 n H
\end{aligned}
$$

Two sets of values will be used in the simulation to check if the whole circuit is really matched at the frequency of operation which is $2.6 \mathrm{GHz}$. Design1 is comprised of $\mathrm{L}_{\mathrm{GS} 1}$ and $\mathrm{L}_{\mathrm{GP} 1}$ for the input matching network and $\mathrm{L}_{\mathrm{LS} 1}$ and $\mathrm{L}_{\mathrm{LP} 1}$ for the output matching network. On the other hand, Design2 is composed of $\mathrm{L}_{\mathrm{GS} 2}$ and $\mathrm{C}_{\mathrm{GP} 2}$ for the input matching network and $\mathrm{L}_{\mathrm{LS} 2}$ and $\mathrm{C}_{\mathrm{LP} 2}$ for the output matching network. The actual values of inductors and capacitors are listed in Table 6. Note that the gain requirement for the amplifier design is set at $10 \mathrm{~dB}$, and hopefully the computed $\mathrm{L}$ and $\mathrm{C}$ for impedance matching networks could help achieve the target.

Table 6: Actual L-network elements

\begin{tabular}{c|c}
\hline $\boldsymbol{L}$ and $\boldsymbol{C}$ & Values \\
\hline$L_{G P 1}$ & $4.186 \mathrm{nH}$ \\
$C_{G P 2}$ & $0.895 \mathrm{pF}$ \\
$L_{G S 1}$ & $5.447 \mathrm{nH}$ \\
$L_{G S 2}$ & $8.364 \mathrm{nH}$ \\
$L_{L P 1}$ & $3.808 \mathrm{nH}$ \\
$C_{L P 2}$ & $0.984 \mathrm{pF}$ \\
$L_{L S 1}$ & $0.311 \mathrm{nH}$ \\
$L_{L S 2}$ & $3.300 \mathrm{nH}$ \\
\hline
\end{tabular}




\section{SIMULATION RESULTS AND ANALYSIS}

Two designs were simulated using the two sets of values of the input and output matching networks, with values previously summarized in Table 6. The actual values are shown in Table 6. Figures 4 and 5 shows the complete schematic circuit designs of Design1 and Design2.

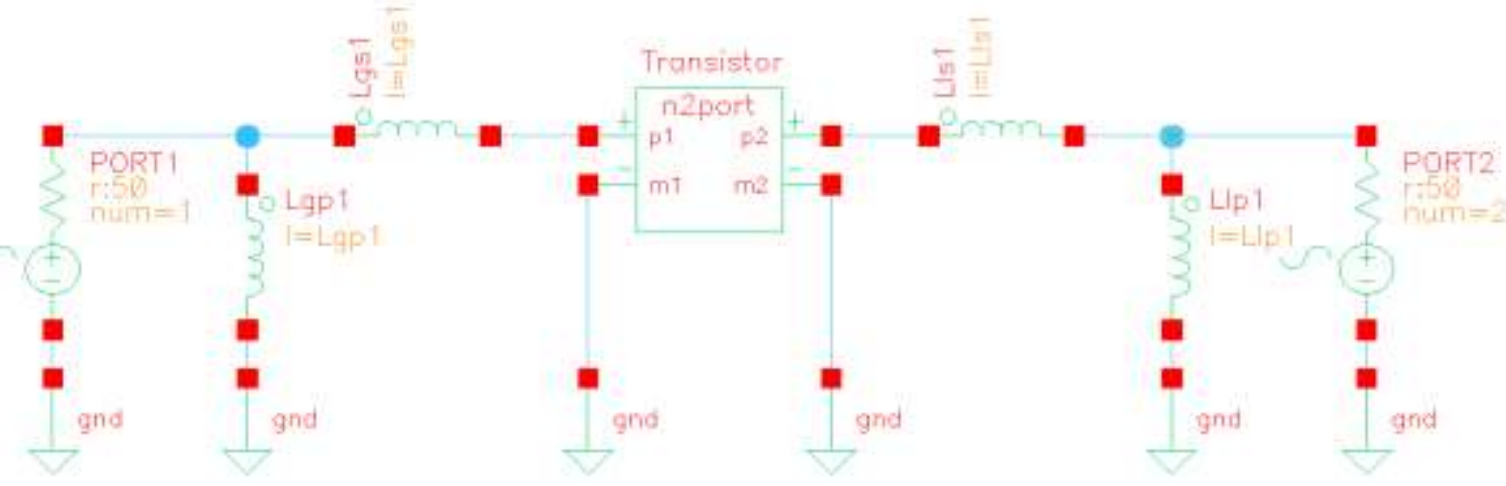

Figure 4: Design1 schematic diagram

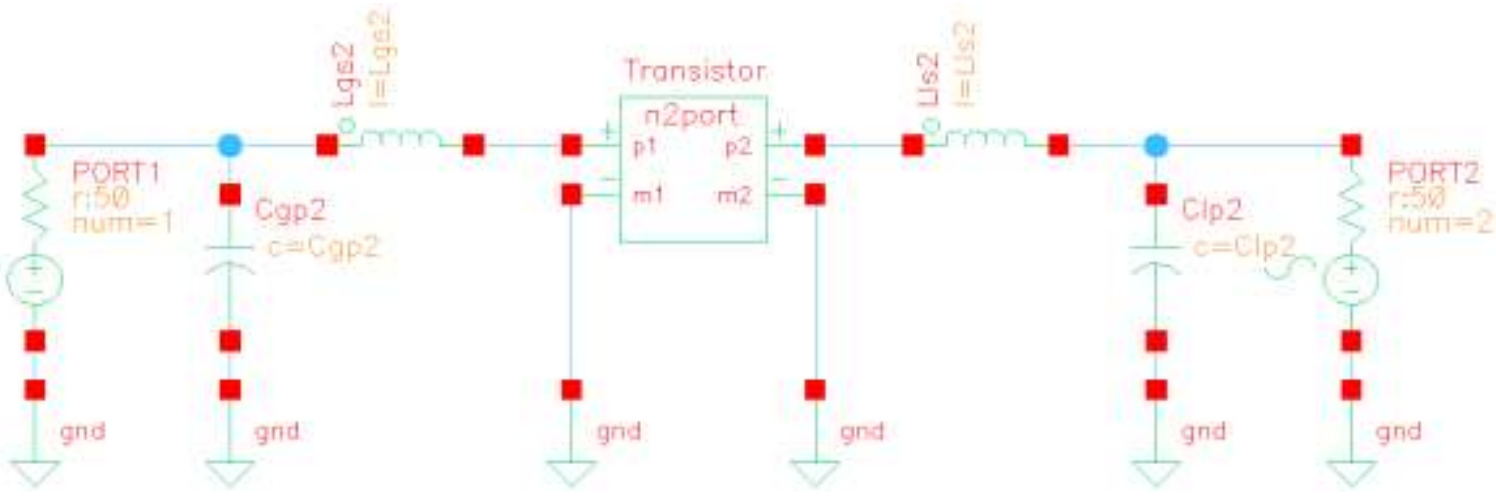

Figure 5: Design2 schematic diagram

The $n 2$ port from the analogLib library is used for the two-port network. Although spectre-format file is preferred for the S-parameter file input of the n2port component, touchstone-format can still be used. In this study, the touchstoneformat S-parameter file is used since the actual S-parameters are given in touchstone format. Still, touchstone-formatted file can be converted to spectre-format using the command sptr. Figures 6 to 9 shows the comparison of the results of the S-parameter plots of the two designs.

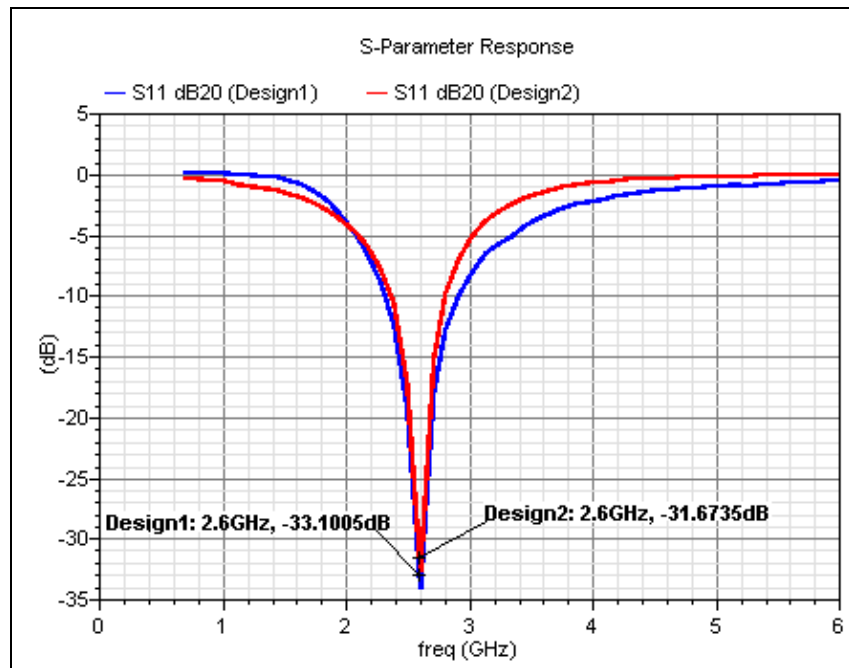

Figure 6. $S_{11}$ plot (in $\mathrm{dB}$ ) versus frequency

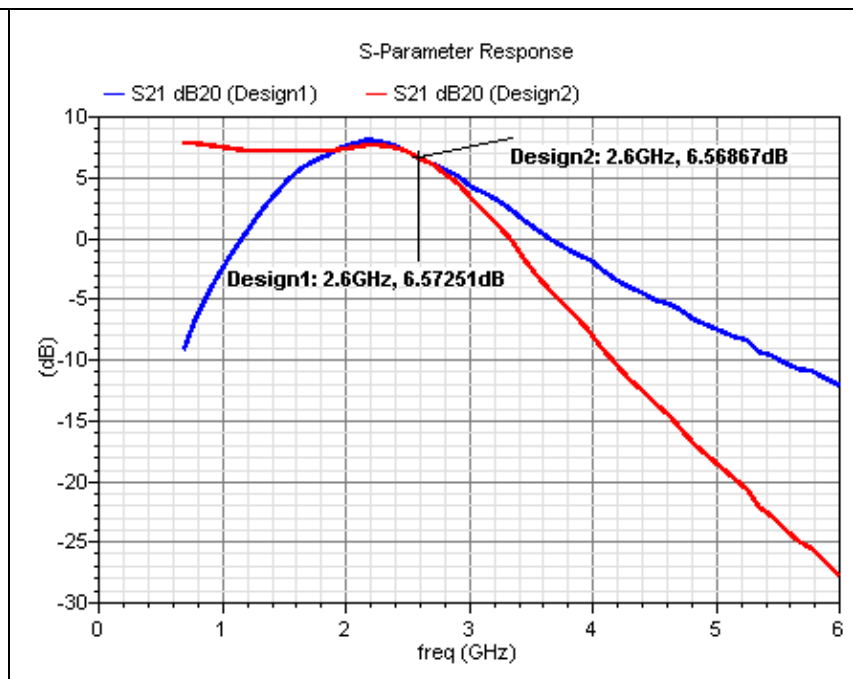

Figure 7. $S_{21}$ plot (in dB) versus frequency 


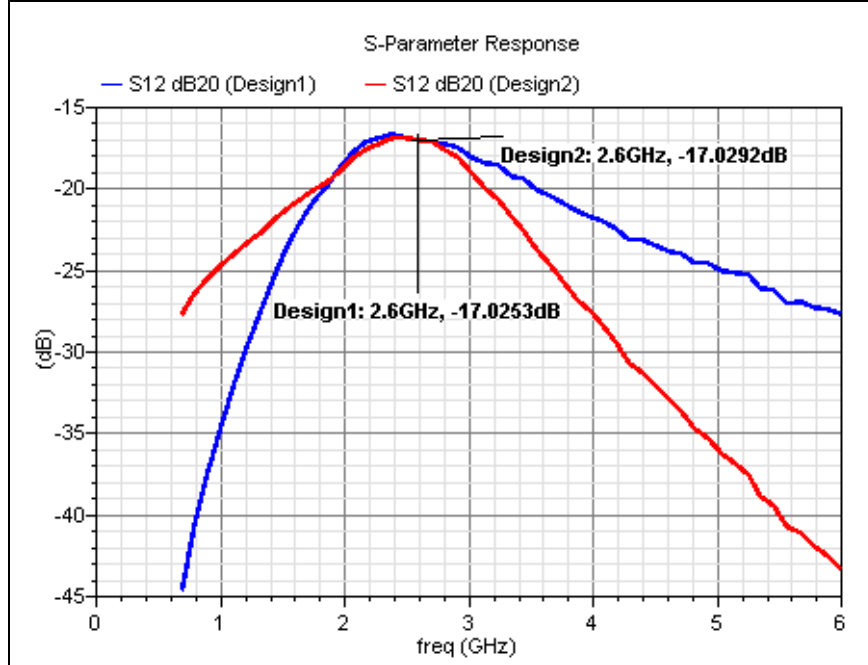

Figure 8. $S_{12}$ plot (in $\mathrm{dB}$ ) versus frequency

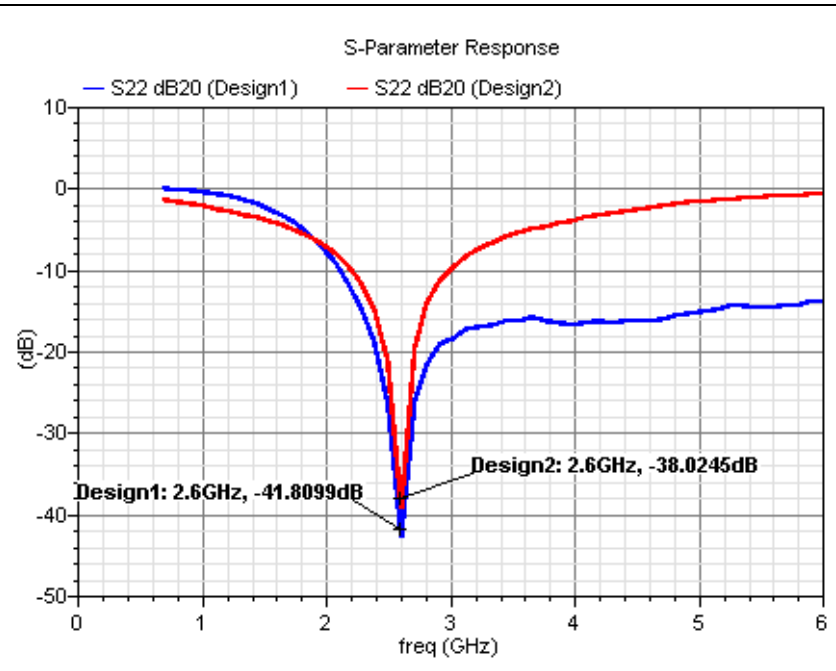

Figure 9. $S_{22}$ plot (in $\mathrm{dB}$ ) versus frequency

S-parameter plots were obtained using the sp analysis. It can be shown in Figure 6 that the two designs are somehow matched at frequency of $2.6 \mathrm{GHz}$. The values of the S-parameters for the two designs at $2.6 \mathrm{GHz}$ are comparable and relatively close to each other. But it can be observed that the S-parameter plots of Design2 are smoother than the plots of Design1 at frequencies greater than $2.6 \mathrm{GHz}$. The difference is evident esp. in the $\mathrm{S}_{22}$ plot in Figure 9 . This signifies that Design2, which is comprised of inductor-capacitor combination in the L-matching networks, exhibits a more stable behavior for higher frequencies than the Design1 which is an all-inductor design. Moreover, the $S_{11}$ and $S_{22}$ plots of Design2 are more symmetric in reference to the frequency of operation which is $2.6 \mathrm{GHz}$ compared to the Design1. A summary of S-parameters values are shown in Table 7.

Table 7: S-parameters response at $2.6 \mathrm{GHz}$

\begin{tabular}{c|r|r}
\hline S-Parameters & Design1 & Design2 \\
\hline$S_{11}$ & $-33.101 \mathrm{~dB}$ & $-31.674 \mathrm{~dB}$ \\
$S_{21}$ & $6.573 \mathrm{~dB}$ & $6.569 \mathrm{~dB}$ \\
$S_{12}$ & $-17.025 \mathrm{~dB}$ & $-17.029 \mathrm{~dB}$ \\
$S_{22}$ & $-41.810 \mathrm{~dB}$ & $-38.025 \mathrm{~dB}$ \\
\hline
\end{tabular}

The gain of the transistor or the amplifier is shown in the $S_{21}$ plot in Figure 7. At frequency of 2.6GHz, the gain is only $6.573 \mathrm{~dB}$ for the Design1 and $6.569 \mathrm{~dB}$ for the Design2. It is almost $3.5 \mathrm{~dB}$ less than the $10 \mathrm{~dB}$ gain target. This is because as the frequency increases in the higher frequencies esp. beyond the frequency of operation, the gain decreases. If the gain-bandwidth product is to be remained constant, then as the bandwidth or the frequency increases, the gain should compensate, thus decreasing the gain. Figures 10 to 15 shows the S-parameter plots in Smith charts.

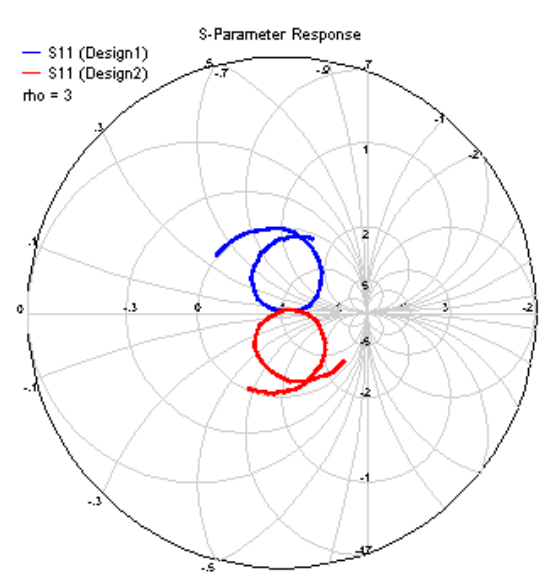

Figure 10. $S_{11}$ impedance Smith chart plot

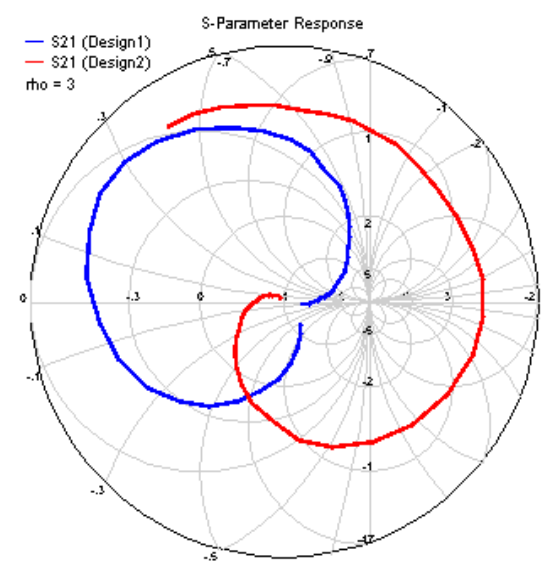

Figure 11. $S_{21}$ impedance Smith chart plot 


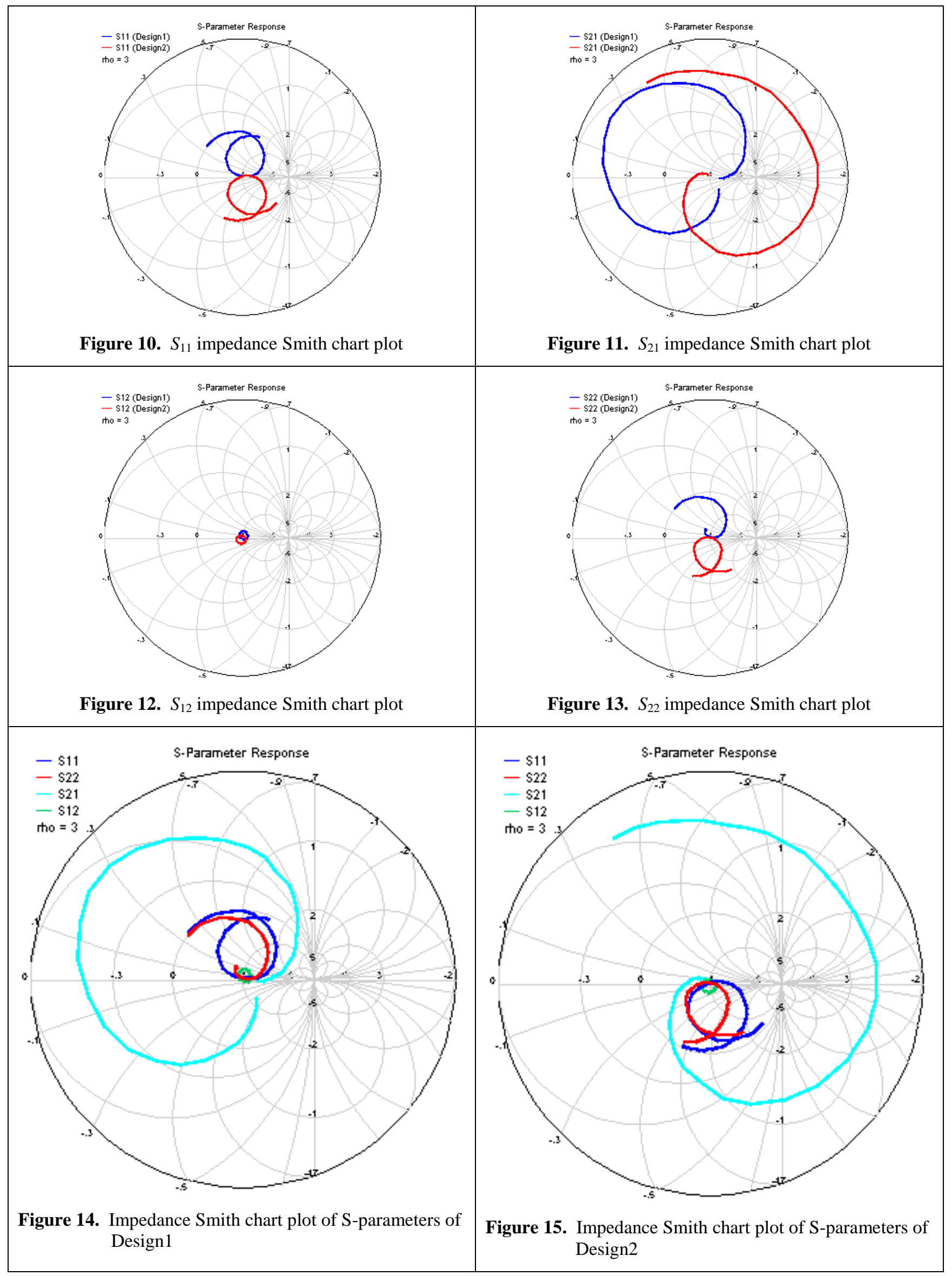


Since Design1 is an all-inductor design, the responses of S-parameters in the impedance Smith chart are more on the inductive half of the Smith chart, evident in Figures 10 to 14. On the other hand, Design2 has a capacitor on the matching networks, thus the impedance Smith chart responses of the S-parameters are more on the capacitive half of the Smith chart as evident in the charts shown in Figures 10-13, 15.

\section{CONCLUSIONS AND RECOMMENDATIONS}

Impedance matching is necessary in RF circuit design to provide maximum possible power transfer between the source or generator and the output load. In this study, two designs were modeled and investigated. The design (Design2) which comprised of an inductor-capacitor combination in the input and output matching networks resulted to a smoother response or a more stable behavior for higher frequencies than the design (Design1) with all inductors in the matching networks. All designs achieved gain of $3 \mathrm{~dB}$ versus the target of $10 \mathrm{~dB}$. One factor is the limitation of the initially provided actual S-parameters of the transistor in touchstone format, which were used for the transistor model using n2port two-port network component. Regardless, complex tradeoffs among technology specifications and design parameters exist and should be carefully handled when designing the impedance matching networks, to optimize the performance of the RF circuit.

Design and study of particular passive components could be helpful in understanding and finally designing the matching networks. Software tools like ASITIC (analysis and simulation of spiral inductors and transformers for ICs) [4] [5] and SpiralCalc (integrated spiral inductor calculator) [6] [7] [8] [9], which are available for non-commercial purposes, could be used for this particular study.

\section{ACKNOWLEDGMENT}

The author would like to express great appreciation to Prof. Maria Theresa De Leon, Ph.D. of Microelectronics and Microprocessors Laboratory at the University of the Philippines for the technical support during the course of the study, and to the STMicroelectronics Calamba NPI Team and the Management Team for the utmost support.

\section{REFERENCES}

[1] C. Bowick, RF Circuit Design, 1st ed., Howard W. Sams \& Co. Inc., Indianapolis, Indiana, USA, 1982.

[2] B. Razavi, RF Microelectronics, 2nd ed., Prentice Hall Press, Upper Saddle River, New Jersey, USA, 2011.

[3] P.R. Gray and R.G. Meyer, "Future directions in silicon ICs for RF personal communications," in Proc. IEEE Custom Integrated Circuits Conference, Santa Clara, CA, USA, pp. 83-89, May 1995.

[4] A.M. Niknejad and R.G. Meyer, "ASITIC for Windows NT/2000," Research in RFIC Design, http://rfic.eecs. berkeley.edu/ niknejad/Asitic/grackle/cygwin_info.html.

[5] A.M. Niknejad and R.G. Meyer, "Analysis and optimization of monolithic inductors and transformers for RF ICs," in Proc. IEEE Custom Integrated Circuits Conference, Santa Clara, CA, USA, pp. 375-378, May 1997.

[6] Stanford Microwave Integrated Circuits Laboratory, Integrated Spiral Inductor Calculator, http://www-smirc. standford.edu/spiralCalc.html.

[7] S.S. Mohan, M. Hershenson, S.P. Boyd and T.H. Lee, "Simple accurate expressions for planar spiral inductances," IEEE Journal of Solid-State Circuits, vol. 34, issue 10, pp. 1419-1424, October 1999.

[8] H.A. Wheeler, "Simple inductance formulas for radio coils," in Proc. Institute of Radio Engineers, vol. 16, issue 10, pp. 1398-1400, October 1928.

[9] M. Hershenson, S.S. Mohan, S.P. Boyd and T.H. Lee, "Optimization of inductor circuits via geometric programming,” in Proc. Design Automation Conference, New Orleans, LA, USA, pp. 994-998, June 1999. 